\title{
Statistical analysis plan for Early mobilisation by head-up tilt with stepping versus standard care after severe traumatic brain injury - a randomised clinical feasibility trial
}

Christian Gunge Riberholt ( $\nabla$ christian.riberholt@regionh.dk )

Rigshospitalet https://orcid.org/0000-0002-6170-1869

Christian Gluud

Copenhagen Trial Unit, Centre for Clinical Intervention Research, Copenhagen University Hospital, Rigshospitalet Janus Christian Jakobsen

Copenhagen Trial Unit, Centre for Clinical Intervention Research, Copenhagen University Hospital, Rigshospitalet

\section{Christian Ovesen}

Department of Neurology, Bispebjerg and Frederiksberg Hospital

\section{Jesper Mehlsen}

Surgical Pathophysiology Unit, Juliane Marie Centre, Copenhagen University Hospital, Rigshospitalet

Kirsten Møller

Department of Neuroanaesthesiology, Rigshospitalet, Copenhagen University

\section{Update}

Keywords:

Posted Date: June 14th, 2019

DOl: https://doi.org/10.21203/rs.2.468/v2

License: (a) This work is licensed under a Creative Commons Attribution 4.0 International License. Read Full License 


\section{Abstract}

Background: Early mobilisation on a tilt table with stepping versus standard care may be beneficial for patients with severe brain injury, but data from randomised clinical trials are lacking. Methods: This statistical analysis plan describes the analyses of data collected in a randomised clinical feasibility trial for early mobilisation by head-up tilt with stepping versus standard care after severe traumatic brain injury. Primary feasibility outcomes are the proportion of included participants who were randomised out of all screened patients; the proportion of participants allocated to the experimental intervention who received at least $60 \%$ of the planned exercise sessions; and safety outcomes such as severe adverse events and reactions and adverse events and reactions. Exploratory clinical outcomes are suspected unexpected severe adverse reactions; and functional outcomes as assessed by Coma Recovery Scale Revised at four weeks; Early Functional Ability Scale, and Functional Independence Measure at three months. Exploratory physiological outcomes are electrocardiographic data; mean arterial pressure; and middle cerebral artery blood flow velocity, all obtained during the head-up tilt test. From the first head-up tilt test and five days onwards, a heart rate was measured by continuous electrocardiography. The detailed description includes the statistical analysis including use of multiple imputation and Trial Sequential Analysis. Conclusions: The present statistical analysis plan serves to minimise potential trial reporting bias and selective $\mathrm{P}$ hacking and to improve transparency. This feasibility trial will inform design and eventual launching of a larger multicentre randomised clinical trial. Trial registration: ClinicalTrials.gov identifier: NCT02924649. Registered on 3 October 2016.

\section{Introduction}

The early mobilisation by Head-Up Tilt with stepping versus standard care after severe Traumatic Brain Injury (HUT-TBI) trial was a randomised clinical trial assessing the feasibility of using a tilt-table with integrated stepping for early mobilisation to the upright position in the neuro-intensive care unit ( 1 ). The possible negative effects of bed rest on human physiology have been investigated for decades (2-4). With the possibility of counteracting the negative effects of prolonged bedrest, it might be beneficial for the patients to undergo early mobilisation whereby they are moved to the upright position using a tilt table. The simultaneous stepping is intended to counteract orthostatic hypotension in the standing position.

Early rehabilitation of patients with severe traumatic brain injury has hitherto been subject to few studies, in which interventions are incompletely described $(5,6)$. Nonetheless, the available studies indicate that functional outcome after traumatic brain injury may be improved by such early rehabilitation.

Furthermore, a large randomised clinical trial, the AVERT trial, showed no benefit of early and intensive mobilisation on poor functional outcomeas measured three months after stroke ( 8 ). Finally, a systematic review with a meta-analysis found no impact of early active mobilisation and rehabilitation on mortality at discharge from the intensive care unit (ICU) in a large variety of non-neurological intensive care unit patients, although the intervention did increase muscle strength, walking ability, and the number of days alive and out of hospital at six months ( 7 ). 
The present trial assessed if using a tilt table for early orthostatic exercise was feasible in a group of patients with severe traumatic brain injury ( 1 ). Here we report the statistical analysis plan for the HUTTBI trial ( 1 ), which has been updated and finalised during the data collection period. Besides the primary outcomes related to feasibility, the analysis plan also addresses the statistical handling of exploratory clinical outcomes and outcomes from sub-studies of cerebral autoregulation and heart rate variability.

\section{Methods}

\section{Ethical approval}

This randomised clinical feasibility trial was approved by the Scientific-Ethics Committee of the Capital Region (H-16041794) and is registered on www.clinicaltrials.gov (ClinicalTrials.gov identifier: NCT02924649); the trial protocol has been published in Trials ( 1 ). The project manager (CGR) is responsible for collecting and storing data and all correspondence. After a patient is found to be eligible for the trial, informed consent from the proxy and a trial guardian (a physician not involved in the trial) is obtained by CGR. CGR is responsible for collecting and storing data and all correspondence. The trial is carried out in accordance with the principles of the Helsinki Declaration ( 9 ).

\section{Primary research questions}

Is an early head-up tilt protocol feasible in patients with severe traumatic brain injury, in terms of the number of participants who are successfully included, number of exercise sessions performed in the experimental group, and the number of patients with serious and non-serious adverse events and adverse reactions?

\section{Exploratory research questions}

Does early head-up tilt with stepping reduce the number of adverse events and reactions (including severe adverse events and reactions) compared with standard care after severe traumatic brain injury?

Does early head-up tilt with stepping improve the level of consciousness (Coma Recovery Scale Revised) after four weeks, early functional abilities (Early Functional Ability scale) after three months, or functional independence (Functional Independence Measure) after three months, compared with standard care after severe traumatic brain injury?

Is cerebral autoregulation of blood flow affected by early head-up tilt with stepping after two and four weeks, compared with standard care after severe traumatic brain injury?

Does head-up tilt with stepping improve the level of consciousness (Coma Recovery Scale - Revised), early functional abilities (Early Functional Ability scale), or functional independence (Functional 
Independence Measure) after one year, compared with standard care in patients with severe traumatic brain injury?

Is there a relationship between specified heart rate variability parameters and cerebral autoregulation of blood flow, orthostatic hypotension, and functional outcome?

\section{Main trial design}

The present statistical analysis plan describes our planned analyses for the feasibility trial, investigating head up tilt with stepping versus standard care in patients with severe traumatic brain injury.

The trial is a randomised clinical feasibility trial with a pragmatic stratification according to the Glasgow Coma Score at inclusion (3-6 compared to 7-10 points). The patients are randomised in a 1:1 ratio by the Copenhagen Trial Unit using a central web-based randomisation system.

Besides standard care, the experimental intervention group receives daily (Monday to Friday) mobilisation on a tilt-table to the standing position for up to 20 minutes per session. This orthostatic exercise continues for four weeks from randomisation or until the patient can stand from a chair or bed with assistance. The tilt-table has a build in stepping device that increases the venous return of blood to the heart and thereby counteracts orthostatic hypotension and increases standing time. The control group receives standard care. Standard care is decided in collaboration between doctors, nurses, and physiotherapists and will be monitored during the trial. Only a small amount of time is used on mobilising the patient to either the edge of the bed or to a wheelchair whilst admitted to the neurologic ICU (NICU). The focus of the physiotherapist is on respiratory function and in bed positioning to avoid bedsores.

Exploratory outcomes are measured using the Coma Recovery Scale - Revised (CRS-R) ( 10 ), the Early Functional Ability (EFA) scale ( 11 ), and the Functional Independence Measure (FIM) $(12,13)$ after four weeks, three months, and one year. The primary time point of assessment for CRS-R is at four weeks while the primary time point is at three months for EFA and FIM.

Additional exploratory physiological outcomes are blood pressure, blood flow velocity of the middle cerebral artery (MCAv), and electrocardiography measured in supine position and during head-up tilt at two weeks and four weeks after randomisation. Furthermore, heart rate variability will be recorded by continuous electrocardiography for up to five days from randomisation in the trial.

\section{Results}

\section{The primary feasibility outcomes}

Our primary feasibility outcomes are: 
The lower limit of the confidence interval of the inclusion ratio (the proportion of included participants randomised compared to all eligible patients). For example, if the number of eligible patients who agree to participate is 44 out of 60 , then the proportion will be $73 \%$ with a $95 \%$ confidence interval $(\mathrm{Cl})$ between $60 \%$ and $84 \%$. The lower limit for this feasibility outcome is set at $60 \%$; if the lower limit of the confidence interval of the gathered data of the HUT-TBI Trial is higher, then the trial is considered to be successful in terms of inclusion.

The lower limit of the confidence interval of the intervention success rate defined as the proportion of participants allocated to the experimental intervention who received at least $60 \%$ of the planned exercise sessions. For example, if the number of participants receiving $60 \%$ of the exercise is $70 \%$ (21 out of 30 ) of the participants randomised to the experimental intervention group, the lower limit of the confidence interval will be $52 \%$. Accordingly, if the lower limit of the confidence interval of the gathered data of the HUT-TBI Trial is above $52 \%$, the trial will be considered to be successful in terms of exercise completeness.

Our safety outcomes are defined as either proportion of participants with serious adverse events (SAEs) and reactions (SARs) or adverse events or adverse reactions not considered serious (AEs or ARs) (14). SAEs are defined as any undesirable event that result in death, is life-threatening, requires prolongation of existing hospitalisation, results in persistent or significant disability or incapacity, or requires intervention to prevent permanent impairment or damage, whether considered related to the trial intervention or not. AEs are defined as any undesirable event not considered serious occurring to a participant during the trial. The proportion of participants with at least one SAE, SAR, AR, or AE during the intervention period will be compared between the two intervention groups. The proportion of serious adverse events will be assessed through inspection and through statistical analysis (see below).

\section{Exploratory clinical outcomes}

For the exploratory clinical outcomes, we have chosen three outcomes: The Coma Recovery Scale Revised (CRS-R), the Early Functional Ability scale (EFA), and the Functional Independence Measure (FIM). The CRS-R reflects changes in consciousness and will be analysed at the four-week time point (end of intervention period) comparing the two intervention groups. The EFA evaluates early functional changes and the FIM evaluates the ability to independently perform functions and activities of daily living. Both will be evaluated at the three-month time point. Secondly, the data for all three exploratory clinical outcomes will be presented as longitudinal data in a figure including confidence intervals. At the one-year follow up, the same three outcome scales are used and supplemented by the Glasgow Outcome Scale - Extended (GOSE), as it is used routinely at the department for the one-year follow-up.

\section{Exploratory physiological outcomes}


For exploratory physiological outcomes we have measured the haemodynamic response to a head-up tilt at baseline, after two weeks, and after four weeks in both groups. From these data we will calculate the cerebral autoregulation index (Mxa) measured by transcranial Doppler ultrasound of the middle cerebral artery at each time point. In short, mean arterial blood pressure and mean middle cerebral artery flow velocity are correlated using a Pearson correlation coefficient. Correlations are calculated during 300 seconds in the supine position (rest) and 300 seconds during head up tilt with 70 degrees head elevation. The Mxa will then principally be considered dichotomously in order to reflect intact cerebral autoregulation of blood flow, with a correlation equal to or below 0.3 or impaired if it is above 0.3 ( 15 ). Data at four weeks will be compared between groups. Secondarily, we will analyse the Mxa as a continuous variable and we will utilise other methods such as the Gosling Pulsatile index, or the cerebrovascular resistance index.

Concerning heart rate variability, we will measure heart rate for five consecutive days from the time of randomisation. A steady period of five minutes before the first head up tilt will be analysed for low frequency, high frequency, and the ratio between low and high frequency both percentage and normalised units (frequency domain) ( 16 ). For comparison between groups, a five minutes interval before the tilt test performed at the $4^{\text {th }}$ week (end of intervention) will be used. Further, a comparison of each head-up tilt test ( 2 weeks and 4 weeks) will be analysed using the same domain parameters for between group comparison as longitudinal analysis. Finally, the frequency domain indices will be used in the attempt to establish a model for predicting the risk of experiencing orthostatic hypotension or impaired cerebral autoregulation at each head-up tilt test.

Afterwards, 24-hour analysis of the heart rate variability will be made within the time domain using the root mean square of successive RR intervals and the standard deviations of NN intervals in normalised units ( 16 ). First, we will analyse the differences between groups at the three-day time point. Then the 24hour analysis will be compared between groups as longitudinal data from the first day of inclusion till day five. Finally, the time domain parameters at the first day after randomisation will be used for a prediction model for clinical outcomes at four-weeks (CRS-R) and at three months (EFA and FIM).

\section{Statistical analyses}

Statistical analysis will be handled using STATA (StataCorp, College Station, Texas, USA).

All baseline characteristics will be presented for each intervention group. Continuous variables will be summarised using means and standard deviations or medians and interquartile range depending on distribution of data. Discrete variables will be presented as frequencies, proportions, and percentages.

Timing of outcome assessments can be found in the published protocol in figure 1 ( 1 ).

All our analyses will primarily be intention-to-treat, i.e. all randomised participants will be included in the primary analyses. We will secondly perform per protocol analyses including the participants allocated to 
the intervention who received at least $60 \%$ of the planned exercise sessions.

If we do not reach the desired number of participants in the trial, we will consider to analyse our data using Trial Sequential Analysis $(17,18)$.

Analysis will start after the last three-month follow-up has been collected and after submission of this statistical analysis plan (end of March 2019). The analysis of the one-year follow-up data will start after data from the last patient has been collected in late December 2019.

\section{Feasibility outcomes}

The first two primary feasibility outcomes will be derived from the trial with the above-mentioned lower limits of the proportions, and these two outcomes will not undergo statistical testing. For the intervention to be feasible, both feasibility outcomes should be achieved.

All analysis described below using general linear regression, logistic regression, or mixed-model linear regression will be adjusted for the protocol specified stratification variable (high or low GCS).

We will use inspection of data to evaluate adverse events due to the low power. Secondly, we will use logistic regression to compare the proportions of participants with one or more SAEs, SARs, Ars, and AEs between the two groups ( 1 ). Accordingly, we will use an alpha of $5 \%$. Each patient with at least one SUSAR during the intervention period will be analysed as exploratory feasibility outcome also using logistic regression analysis. Where appropriate we will present data with a $95 \%$ confidence interval.

\section{Exploratory clinical outcome}

All exploratory clinical outcomes and physiological outcomes are on a continuous interval scale.

The exploratory clinical outcomes will primarily be compared between groups at specified time points. The CRS-R will be analysed at the four-week time point and EFA and FIM will be analysed at the threemonth time point using general linear regression analysis. Secondarily, as a sensitivity analysis, longitudinal data will be analysed using mixed model linear regression with each participates as a random effect and the clinical outcome as the fixed effect for analysis of longitudinal data over multiple time points.

Each outcome, the outcomes minimal relevant difference, standard deviation and power level can be found in Supplementary table 1. The one-year follow up data for CRS-R, EFA, and FIM will be analysed in the same way only the three-month time-point will be replaced with one-year. Furthermore, for the one-

year analysis the Glasgow outcome scale extended will be compared between groups using general linear regression and adjusting for stratification specific variables. 
In case the regression models described above (linear regression and mixed model) cannot be fitted due to breach of their underlying assumptions (e.g. skewed distribution of data/residuals), non-parametric methods (e.g. Van Elteren test) taking the stratified randomisation into account will be employed. Analysis will in all cases be conducted at the prespecified time points stipulated above. As described in our protocol, $P$-values of any size will not be interpreted as significant due to the low power of the trial.

\section{Exploratory physiological outcomes}

The exploratory physiological outcomes measuring cerebral autoregulation will be compared at the 4week time point (or end of intervention). To do this, we will use logistic regression with the Mxa (binary) as the dependent and further adjusting for age and sex. The Mxa will also be tested as a continuous variable (ranging from -1 to 1) using general linear regression analysis and mixed-model linear regression with each participant as a random effect for analysis of longitudinal data over multiple time points. The same approach will be used for the Gosling Pulsatile index and the cerebrovascular resistance index.

It is our intention to analyse and publish data on the heart rate variability frequency domain and time domain indices. The statistical analysis plan for these outcomes will be made public in a separate report.

\section{Missing data}

Trials conducted in the ICU are at high risk of missing data alone on the account of the patient's condition ( 19 ). If data are missing, we will consider using multiple imputation according to the recommendations by Jakobsen and colleagues ( 20 ). For all continuous clinical outcomes, we will analyse survivors, and in a sensitivity analysis impute the lowest possible value for participants who died or dropped out as well as the best possible value. We will present the results of both analyses.

\section{Trial status and profile}

The inclusion period ended in December 2018 with only 38 patients included during a two-year period. End of the three-month follow-up period will be in March 2019 and the one-year follow up will be in December 2019. Flow of patients will be presented in a CONSORT diagram as reported in the protocol ( 1 ). We will report the number of screened patients, the number of included patients, and the main reason for exclusion of eligible patients. Furthermore, we will present the number of patients who died within the four-week intervention period, within the first three months from randomisation, and within the first year.

\section{Publication strategy}

We plan to publish the following papers: 
The primary feasibility outcomes and the analyses of the exploratory clinical outcomes will be published separately to establish feasibility of the intervention and the sample size of a larger multicentre study (publication I).

Analysis of the exploratory outcomes with the focus of investigating a specific physiological effect on cerebral autoregulation after a series of orthostatic exercises during head up tilt (publication II).

Analysis of one-year follow-up data once all data are collected, which is expected to be in December 2019 (publication III).

\section{Presentation of results in tables and figures}

Publication I: The CONSORT flow chart diagram will be presented as Figure 1. To assess the balance of our randomisation, description of baseline characteristics will be presented in a table (Table 1). Variables will be summarized as either frequencies and percentages or as continues or ordinal variables as mean with standard deviations (SD) or median and interquartile range (IQR) (see Appendix 1).

The primary feasibility outcome will be presented in Table 2 and Table 3 . The first table will consist of the proportion of patients included in the trial as well as the number of patients who successfully received more than $60 \%$ of the intended interventions. Table 3 will present and describe the adverse events seen during the intervention period as well as the logistic regression analysis.

The exploratory clinical outcome will be presented as shown in Table 4, with the absolute numbers of each outcome at each time point in a Supplementary table 2. Furthermore, the longitudinal analysis will be presented in a figure showing each time point with confidence intervals (not illustrated).

Publication II: The second publication will present Table 1 as a demographic table. The results will be presented in a figure with included table (Figure 2). The figure will consist of three graphs (A, B and C) with data summarized beneath, showing heart rate (HR, mean arterial blood pressure (MAP), and middle cerebral artery blood flow velocity (MCAVmean) $\Delta$-values between supine position and during head up tilt at baseline, after 2 weeks and after 4 weeks. As supplementary material we will present a spaghetti-plot of each of the above variables in supine and in standing (Supplementary figure 1). Table 5 shows the Mxa values in supine and in standing for both groups at the end of the intervention period. Alongside this, a Figure 2 will illustrate the time line for Mxa, with Mxa on the $y$-axis and time on the $\mathrm{x}$-axis, showing a mean with confidence intervals for each time point (baseline, 2 weeks, 4 weeks). Figure 2A will illustrate $M x a$ in the supine and Figure 2B in standing. Lastly, a Table 6 will show the frequency of intact cerebral autoregulation in the two groups with $P$-values indicating group differences.

Publication III: As in publication I this publication will analyse the outcomes after one year. Similarly, the data will be presented as Table 1 and Table 4 with the added 1-year data on each outcome as well as Glasgow outcome scale extended at the 1-year follow-up. 


\section{Discussion}

This statistical analysis plan for the feasibility trial of conducting early orthostatic exercise in patients with severe traumatic brain injury is published to minimise outcome reporting bias and data-driven results. From the total data gathered in the study, the primary outcomes are feasibility outcomes, but we have also described assessments of our exploratory outcomes.

Our statistical analysis plan is based on considerations to secure unbiased data handling and analyses without getting inspired by the collected data, i.e. P-hacking ( 17 ).

Our statistical analysis plan has several limitations. The primary limitation is that the trial was stopped early. The sample size in this trial was pragmatically set to 60 participants with severe traumatic brain injury, but we only randomised 38 participants. This is a major limitation of this trial. Especially the analyses of the adverse events and the exploratory outcomes will be underpowered and should be considered preliminary and hypothesis generating only. Furthermore, multiple imputation for missing data assumes that these are missing at random; however, this assumption may be incorrect. For example, data completeness may differ between patients in the intervention and the control group.

Our primary clinical outcomes, especially the CRS-R and EFA, could have been subject to more thorough testing, as no published minimal real difference values existed for these outcomes. Therefore, the power of the current study is uncertain.

\section{Conclusions}

The HUT-TBI trial investigates the feasibility of early orthostatic exercise. With the present pre-specified statistical analysis plan, we hope to minimise analytic bias. On the the larger scale, we hope that the feasibility outcomes and the exploratory outcomes may inform and enable generation of hypotheses for a larger multicentre trial investigating benefits and harms of early orthostatic exercise.

\section{Abbreviations}

AE: adverse event; AR: adverse reaction; CG: Control group; CI95\%: 95\% confidence interval; CRS-R: Coma Recovery Scale - Revised; CVRI: Cerebrovascular Resistance Index; EFA: Early Functional Ability; EOE: Early orthostatic exercise; FIM: Functional Independence Measure; GCS: Glasgow coma scale; GPI: Gosling's Pulsatile Index; HR: heart rate; ICU: Intensive care unit; MAP: Mean arterial pressure; MCAv: Middle cerebral artery blood flow velocity; Mxa: cerebral autoregulation index; NICU: Neuro intensive care unit; SAR: serious adverse reaction; SAE: serious adverse event; SD: standard deviation; SUSAR: suspected unexpected adverse reaction;

\section{Declarations}

\section{Acknowledgements}




\section{Funding}

The trial has been funded by "The Council of Danish Victims Fund" (grant 16-910-00043), by the "Research Fund of Rigshospitalet, Copenhagen University Hospital" (R114-A4672), and the "Danish Physical Therapy Association" (15242). The funders had no influence on the design of the trial.

\section{Availability of data and materials}

The datasets analysed during the current study are not publicly available due to the small sample size, but are available from the corresponding author on reasonable request.

\section{Authors' contributions}

CGR drafted the statistical analysis plan. CG, JCJ, JM and KM provided input for drafting and finalising the statistical analysis plan. JCJ acted as senior statistician and CO as co-statistician. JCJ and CO did the analysis independently. KM is the chief investigator of the trial. All authors read and approved the manuscript for publication.

\section{Consent for publication}

Not applicable

\section{Competing interests}

The authors declare that they have no competing interests.

\section{Data management plan and standard operating procedure}

The data management plan and standard operating procedure are kept at the Copenhagen Trial Unit.

\section{References}

[1] Riberholt CG, Lindschou J, Gluud C, Mehlsen J, Moller K. Early mobilisation by head-up tilt with stepping versus standard care after severe traumatic brain injury - Protocol for a randomised clinical feasibility trial. Trials. 2018;19(1):612. 
[2] Saltin B, Blomqvist G, Mitchell JH, Johnson RL, Jr., Wildenthal K, Chapman CB. Response to exercise after bed rest and after training. Circulation. 1968;38(5 Suppl):VII1-78.

[3] Grenon SM, Hurwitz S, Xiao X, Sheynberg N, Ramsdell CD, Kim C, Cohen RJ, Williams GH.

Readaptation from simulated microgravity as a stimulus for improved orthostatic tolerance: role of the renal, cardioendocrine, and cardiovascular systems. J Investig Med. 2005;53(2):82-91.

[4] Allen C, Glasziou P, Del Mar C. Bed rest: a potentially harmful treatment needing more careful evaluation. Lancet. 1999;354(9186):1229-33.

[5] Andelic N, Bautz-Holter E, Ronning P, Olafsen K, Sigurdardottir S, Schanke AK, Sveen U, Tornas S, Sandhaug M, Roe C. Does an early onset and continuous chain of rehabilitation improve the long-term functional outcome of patients with severe traumatic brain injury? J Neurotrauma. 2012;29(1):66-74.

[6] DeJong G, Hsieh CH, Putman K, Smout RJ, Horn SD, Tian W. Physical therapy activities in stroke, knee arthroplasty, and traumatic brain injury rehabilitation: their variation, similarities, and association with functional outcomes. Phys Ther. 2011;91(12):1826-37.

[7] Tipping CJ, Harrold M, Holland A, Romero L, Nisbet T, Hodgson CL. The effects of active mobilisation and rehabilitation in ICU on mortality and function: a systematic review. Intensive Care Med. 2017;43(2):171-83.

[8] Bernhardt J, Langhorne P, Lindley R, Thrift A, Ellery F, Collier J, Churilov L, Moodie M, Dewey H, Donnan G. Efficacy and safety of very early mobilisation within $24 \mathrm{~h}$ of stroke onset (AVERT): a randomised controlled trial. Lancet. 2015;386:46-55.

[9] WMA Declaration of Helsinki - ethical principles for medical research involving human subjects. . 2013; Available from: https:// www.wma.net/policies-post/wma-declaration-of-helsinki-ethical-principlesfor-medical-research-involving-human-subjects/ .

[10] Giacino JT, Kalmar K, Whyte J. The JFK Coma Recovery Scale-Revised: measurement characteristics and diagnostic utility. Arch Phys Med Rehabil. 2004;85(12):2020-9.

[11] Hankemeier A, Rollnik JD. The Early Functional Abilities (EFA) scale to assess neurological and neurosurgical early rehabilitation patients. BMC Neurol. 2015;15:207.

[12] van Baalen $B$, Odding $E$, van Woensel MP, Roebroeck ME. Reliability and sensitivity to change of measurement instruments used in a traumatic brain injury population. Clin Rehabil. 2006;20(8):686-700.

[13] Beninato M, Gill-Body KM, Salles S, Stark PC, Black-Schaffer RM, Stein J. Determination of the minimal clinically important difference in the FIM instrument in patients with stroke. Arch Phys Med Rehabil. 2006;87(1):32-9. 
[14] ICH Harmonised Guideline. Integrated addendum to ICH E6(R1). Guideline for Good Clinical Practice E6(R2), (2015).

[15] Zeiler FA, Donnelly J, Calviello L, Menon DK, Smielewski P, Czosnyka M. Pressure Autoregulation Measurement Techniques in Adult Traumatic Brain Injury, Part I: A Scoping Review of Intermittent/SemiIntermittent Methods. J Neurotrauma. 2017;34(23):3207-23.

[16] Shaffer F, Ginsberg JP. An Overview of Heart Rate Variability Metrics and Norms. Front Public Health. 2017;5:258.

[17] Wetterslev J, Jakobsen JC, Gluud C. Trial Sequential Analysis in systematic reviews with metaanalysis. BMC Med Res Methodol. 2017;17(1):39.

[18] Jakobsen JC. Systematic reviews of randomised clinical trials examining the effects of psychotherapeutic interventions versus "no intervention" for acute major depressive disorder and a randomised trial examining the effects of "third wave" cognitive therapy versus mentalization-based treatment for acute major depressive disorder. Dan Med J. 2014;61(10):B4942.

[19] Bartolo M, Bargellesi S, Castioni CA, Intiso D, Fontana A, Copetti M, Scarponi F, Bonaiuti D.

Mobilization in early rehabilitation in intensive care unit patients with severe acquired cerebral injury: An observational study. J Rehabil Med. 2017.

[20] Jakobsen JC, Gluud C, Wetterslev J, Winkel P. When and how should multiple imputation be used for handling missing data in randomised clinical trials - a practical guide with flowcharts. BMC Med Res Methodol. 2017;17(1):162.

[21] Frazzitta G, Zivi I, Valsecchi R, Bonini S, Maffia S, Molatore K, Sebastianelli L, Zarucchi A, Matteri D, Ercoli G, et al. Effectiveness of a Very Early Stepping Verticalization Protocol in Severe Acquired Brain Injured Patients: A Randomized Pilot Study in ICU. PLoS One. 2016;11(7):e0158030.

[22] Krewer C, Luther M, Koenig E, Muller F. Tilt Table Therapies for Patients with Severe Disorders of Consciousness: A Randomized, Controlled Trial. PLoS One. 2015;10(12):e0143180.

[23] Riberholt CG, Olesen ND, Thing M, Juhl CB, Mehlsen J, Petersen TH. Impaired cerebral autoregulation during head up tilt in patients with severe brain injury. PLoS One. 2016;11(5):e0154831.

[24] Riberholt CG, Thorlund JB, Mehlsen J, Nordenbo AM. Patients with severe acquired brain injury show increased arousal in tilt-table training. Dan Med J. 2013;60(12):A4739.

\section{Tables}

Table 1. Baseline characteristics of included patients 


\begin{tabular}{|l|l|l|}
\hline & Early orthostatic exercise (n=) & Control intervention (n=) \\
\hline Age (years) & & \\
\hline Male - $\mathrm{n}(\%)$ & & \\
\hline $\begin{array}{l}\text { GCS at inclusion - median (IQR) } \\
\text { Low GCS (3-6) - n (\%) } \\
\text { High GCS (7-10) - n (\%) }\end{array}$ & & \\
\hline Brain injury - n (\%) & & \\
aSDH & & \\
EDH & & \\
Cont. & & \\
Etc. & & \\
\hline Mechanism of injury - n (\%) & & \\
Fall & & \\
Traffic (car) & & \\
Etc. & & \\
\hline Secondary injury - n (\%) & & \\
Type of injury & & \\
Etc. & & \\
\hline Neurosurgical procedures performed - n (\%) & & \\
Type of procedure & & \\
Etc. & & \\
\hline GCS at admission - median (IQR) & & \\
\hline Days from injury to randomisation - mean \pm SD & & \\
\hline Sedated at time of intervention - $\mathrm{n}(\%)$ & & \\
\hline RASS at inclusion - median (IQR) & & \\
\hline IQR: Interquartile range; n: number; aSDH: Acute subdural haematoma; EDH: Epidural haematoma; Cont.: \\
Contusion; SD: Standard deviation; GCS: Glasgow coma score; RASS: Richmond agitation sedation scale.
\end{tabular}

\section{Table 2. Characteristics of feasibility outcome}

\begin{tabular}{|l|l|}
\hline & $\mathrm{n} / \mathrm{N}(\%)$ with 95\% confidence interval \\
\hline Included patients per eligible patient & \\
\hline Proportion of patients with more than 60\% completed exercises & \\
\hline
\end{tabular}

\section{Table 3. Adverse events and reactions}

\begin{tabular}{|l|l|l|}
\hline Patients experiencing at least one: & Early orthostatic exercise (n=) & $\begin{array}{l}\text { Control intervention } \\
(\mathrm{n}=)\end{array}$ \\
\hline AE - $\mathrm{n}(\%)$ & & \\
\hline SAE- $\mathrm{n}(\%)$ & & \\
\hline AR- $(\%)$ & & \\
\hline SAR- $\mathrm{n}(\%)$ & & \\
\hline $\begin{array}{l}\text { n: Number; AE: Adverse event; SAE: Serious adverse event; AR: Adverse reaction; SAR: Serious adverse } \\
\text { reaction; P-value calculated using Fishers exact test. }\end{array}$ \\
\hline
\end{tabular}


Table 4. Exploratory clinical outcome at three months

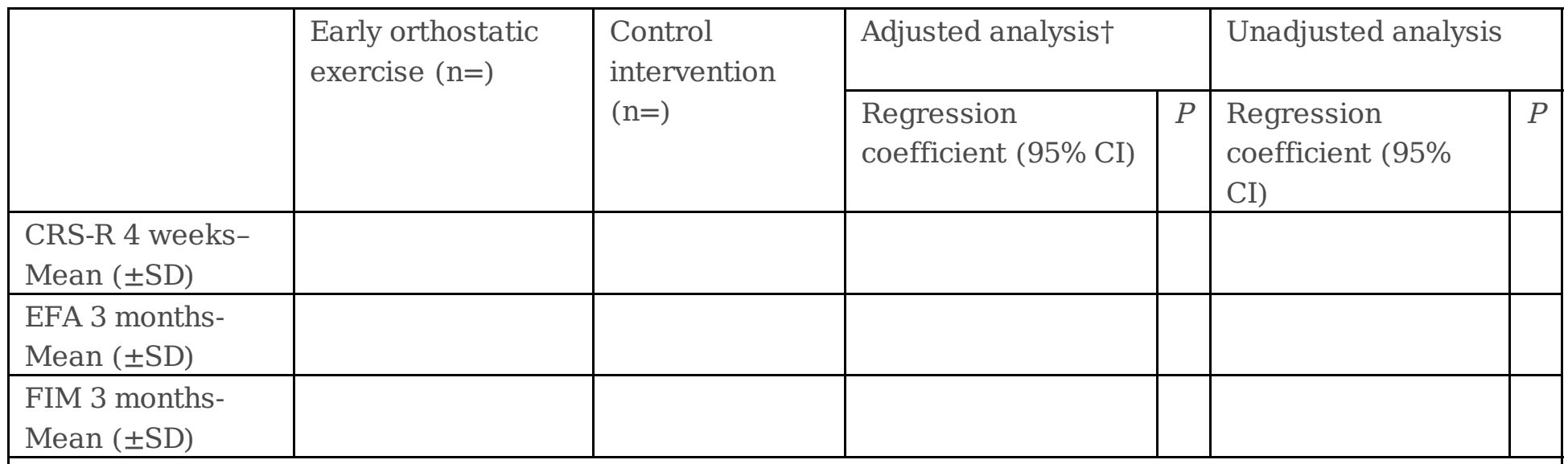

CI 95\%: 95\% confidence interval; CRS-R: Coma Recovery Scale - Revised; EFA: Early Functional Ability; FIM:

Functional Independence Measure; † Adjusted for stratification variable (GCS)

Table 5. Exploratory physiological outcome on autoregulation of cerebral blood flow after 4 weeks inclusion.

\begin{tabular}{|c|c|c|c|c|c|c|}
\hline & \multirow{2}{*}{$\begin{array}{l}\text { Control } \\
\text { intervention } \\
(\mathrm{n}=)\end{array}$} & \multirow{2}{*}{$\begin{array}{l}\text { Early orthostatic } \\
\text { exercise }(n=)\end{array}$} & \multicolumn{2}{|l|}{ Adjusted analysis $\dagger$} & \multicolumn{2}{|l|}{ Unadjusted analysis } \\
\hline & & & $\begin{array}{l}\text { Regression } \\
\text { coefficient (95\% CI) }\end{array}$ & $P$ & $\begin{array}{l}\text { Regression } \\
\text { coefficient (95\% CI) }\end{array}$ & $P$ \\
\hline $\begin{array}{l}\text { Mxa } \\
\text { (supine) } \\
\text { Mean } \\
( \pm S D)\end{array}$ & & & & & & \\
\hline $\begin{array}{l}\text { Mxa (70 } \\
\text { degrees) } \\
\text { Mean } \\
( \pm S D)\end{array}$ & & & & & & \\
\hline $\begin{array}{l}\text { GPI Mear } \\
( \pm S D)\end{array}$ & & & & & & \\
\hline $\begin{array}{l}\text { RI Mean } \\
( \pm S D)\end{array}$ & & & & & & \\
\hline
\end{tabular}

Table 6. Intact cerebral autoregulation at end of intervention. 


\begin{tabular}{|c|c|c|c|c|c|c|}
\hline & Early orthostatic & Control & Adjusted analysis & & Unadjusted analy & \\
\hline & & $(n=)$ & $\begin{array}{l}\text { Regression } \\
\text { coefficient (95\% } \\
\text { CI) }\end{array}$ & $P$ & $\begin{array}{l}\text { Regression } \\
\text { coefficient (95\% } \\
\text { CI) }\end{array}$ & $P$ \\
\hline $\begin{array}{l}\text { Intact cerebral } \\
\text { autoregulation (Mxa } \\
\text { supine) }\end{array}$ & & & & & & \\
\hline $\begin{array}{l}\text { Intact cerebral } \\
\text { autoregulation (Mxa tilt) }\end{array}$ & & & & & & \\
\hline
\end{tabular}

\section{Figures}

Figure 1 not provided with this manuscript version

Figure 1 

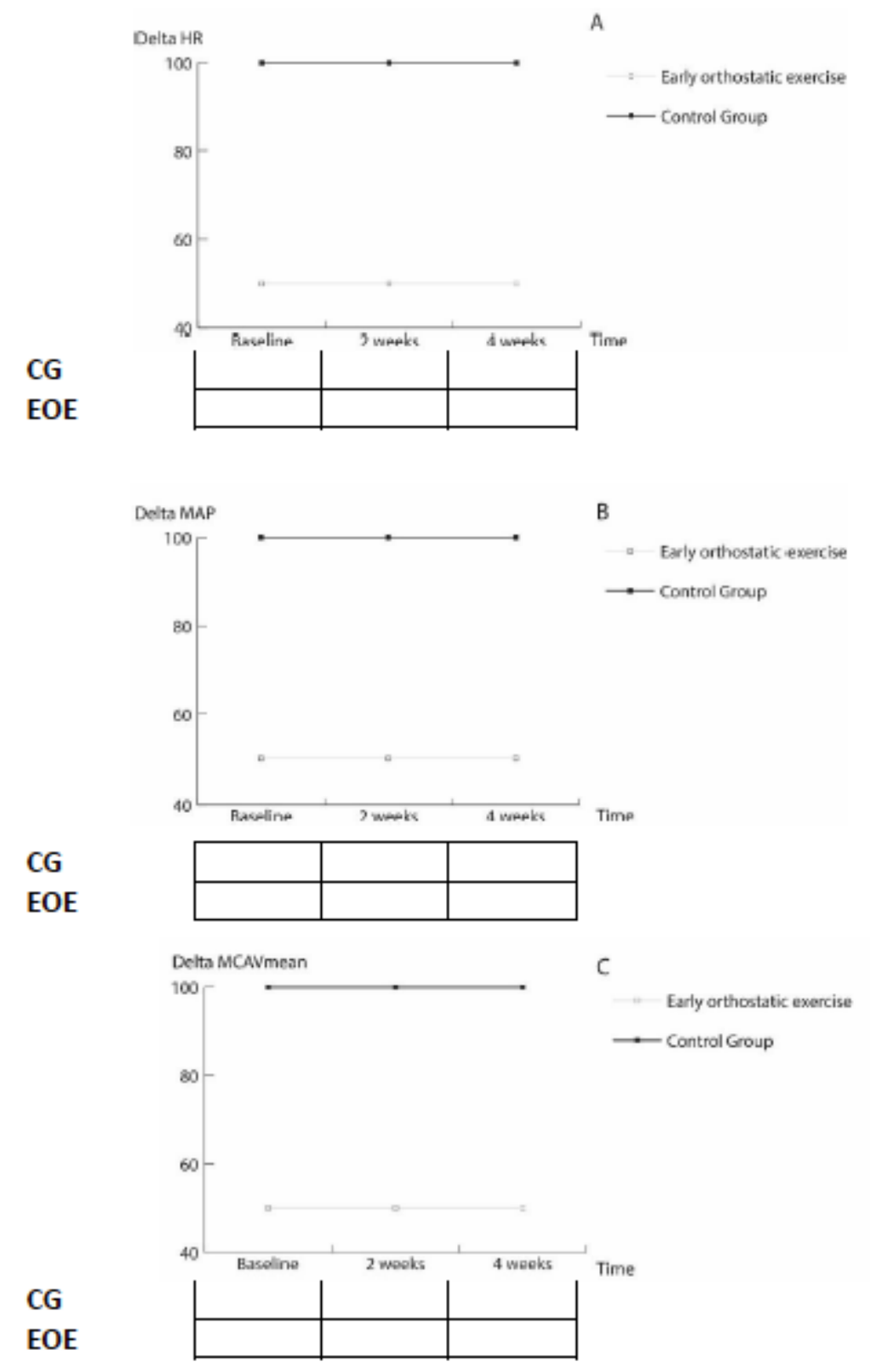

Figure 2

$A, B$, and $C$ including table. Haemodynamic change from supine position to head up tilt. HR: Heart rate; CG: Control group; EOE: Early orthostatic exercise; MAP: Mean arterial pressure; MCAVmean: Mean middle cerebral artery blood flow velocity.

\section{Supplementary Files}

This is a list of supplementary files associated with this preprint. Click to download.

- supplement1.docx

- supplement2.docx

- supplement3.docx 